Rev Inv Vet Perú 2014; 25(4): 449-454

http://dx.doi.org/10.15381/rivep.v25i4.10778

Artículo de Revisión

\title{
Optimización del Rendimiento Deportivo en Caninos
}

\author{
Optimizing Sport Performance in Dogs
}
Francisco Javier Pellegrino ${ }^{1,2}$, Analía Lorena Risso ${ }^{1,2}$, Daniel Osvaldo Arias ${ }^{1}$, Paula Graciela Blanco ${ }^{1,2}$, Yanina Corrada ${ }^{1,2,3}$

\section{Resumen}

El desempeño físico del perro depende de factores genéticos, de entrenamiento y nutricionales. El entrenamiento y la nutrición pueden ser modificados fácilmente. Se deben instaurar programas de ejercicio y dietas que permitan maximizar el rendimiento en una determinada actividad. Para evaluar la condición física de los perros y obtener el máximo rendimiento se necesitan parámetros objetivos como son la observación de las variaciones de la frecuencia cardíaca y la determinación de lactato sanguíneo. Esta última se ha mostrado como un método apropiado, práctico y sencillo que contribuye a cuantificar la respuesta metabólica al esfuerzo. En la actualidad el uso de cinta trotadora motorizada permite llevar a cabo rutinas de entrenamiento específicas y a pesar de que aún restan mayores estudios, su aplicación en caninos tiene un lugar prometedor para maximizar el rendimiento deportivo.

Palabras clave: rendimiento deportivo, canino, entrenamiento, nutrición

\section{Abstract}

Canine physical performance depends on genetic, training and nutritional factors. Training and nutrition can be easily modified. Exercise and diet programs must be established in order to maximize performance on a given activity. To evaluate physical condition of dogs and get maximum performance, objective

\footnotetext{
${ }^{1}$ Facultad de Ciencias Veterinarias, Universidad Nacional de La Plata, Bs As, Argentina

${ }^{2}$ Consejo Nacional de Investigaciones Científicas y Técnicas (CONICET), Argentina

${ }^{3}$ E-mail: y.corrada@gmail.com
} 
parameters such as the observation of variations in heart rate and determination of blood lactate must be established. The latter has been shown as an appropriate, practical and simple method that contributes to quantify the metabolic response to effort. Nowadays the use of motorized treadmills allows to perform specific training routines and, although further studies are required, its application in canines has a promising place to maximize sports performance.

Key words: sports performance, canine, training, nutrition

\section{Introducción}

El deporte canino ha ido creciendo y cobrando gran popularidad en los últimos años. La diversidad de razas y su amplio espectro de habilidad atlética permite que se adapten a diversas actividades de trabajo, caza y deporte (Palmer, 1994). El desempeño físico depende de factores genéticos, de entrenamiento y nutricionales. La constitución genética del animal limitará siempre sus capacidades fisiológicas y funcionales (Grandjean et al., 2000); sin embargo, con el adecuado entrenamiento y nutrición se puede lograr maximizar el rendimiento deportivo.

El entrenamiento deportivo en el canino comprende un proceso continuo de trabajo cuyo objetivo final es maximizar las capacidades físicas, técnico-tácticas y psíquicas, mediante la realización de ejercicios sistemáticos y progresivos, planificados individualmente. Este proceso debe incluir, además, la relación hombre-perro, que mantiene la motivación del animal mediante el aspecto lúdico del esfuerzo que se le exige, logrando así una mayor predisposición $\mathrm{y}$, consecuentemente, una mejor respuesta (Grandjean et al., 2000). De este modo, y con el fin de prevenir la presentación de patologías vinculadas al ejercicio que pueden comprometer el futuro deportivo del animal, propietarios, entrenadores y veterinarios necesitan de parámetros objetivos para poder evaluar la condición física de los perros (Ferasin y Marcora, 2009).

Dentro de los parámetros medibles para calcular la intensidad requerida durante un periodo de trabajo, se encuentran las variaciones de la frecuencia cardíaca (FC) y la determinación de lactato sanguíneo (LS). Se ha demostrado en humanos que la capacidad de metabolizar o tolerar el lactato y producir energía simultáneamente, puede ser un importante indicador de rendimiento aeróbico (Date et al., 2013). La concentración de LS puede determinarse mediante el uso de analizadores enzimáticos portátiles (Ferasin et al., 2007); de este modo, su medición durante el entrenamiento en cinta trotadora motorizada determina el límite del rendimiento aeróbico individual, conocido como umbral anaeróbico, que junto a la evaluación de la FC, permite regular la intensidad de un programa de entrenamiento (García del Moral et al., 1998).

Otro aspecto importante para el éxito deportivo es la correcta nutrición del atleta canino. Los requerimientos energéticos diarios varían según la raza, peso corporal, condición fisiológica, ejercicio y temperatura ambiente (Hill, 1998). En promedio, los valores de mantenimiento se hallan en $132 \mathrm{kcal}$ de Energía Metabolizable [EM]/kg Peso Metabólico (PM = Peso vivo ${ }^{0.73}$ ) (Grandjean et al., 2000). La actividad física induce un gasto energético que es ciertamente mayor al necesario para el mantenimiento diario. Por ejemplo, para esfuerzos de intensidad y tiempo moderado se recomienda entre 200 y 400 $\mathrm{kcal} \mathrm{EM} / \mathrm{kg}^{0.73}$, en tanto que para una actividad de máxima velocidad y corto tiempo es aconsejable aportar entre 150 y $190 \mathrm{kcal} \mathrm{EM/}$ $\mathrm{kg}^{0.73}$ (Grandjean et al., 2000). 
Así, la intensidad y duración determinan el tipo de sistema y la integración metabólica que predominará durante el esfuerzo (McArdle et al., 2004). De esta manera, el sistema aeróbico predomina en ejercicios de baja intensidad en el que las grasas son el combustible principal. Por otra parte, en el ejercicio anaeróbico los carbohidratos contribuyen a la producción de energía, de allí la importancia de conocer los componentes energéticos específicos para cada actividad (McArdle et al., 2004).

El presente documento hace una revisión de los factores y parámetros más importantes que influencian positivamente el rendimiento deportivo en caninos.

\section{Lactato Muscular: Importancia y Pro- ducción durante el Esfuerzo}

El lactato es un producto del metabolismo anaeróbico. Su aparición en sangre está en función de varios mecanismos que intervienen en su producción, consumo y distribución por los distintos compartimientos del organismo, especialmente muscular, sanguíneo e intersticial (García del Moral et al., 1998). Durante muchos años fue considerado como un metabolito de desecho de la glucólisis generado ante isquemias o durante ejercicios de máxima intensidad, nocivo para la célula. Hoy se conoce no solo que es inocuo, sino que además tiene efectos benéficos para el organismo como ser metabolito intermediario en la gluconeogénesis hepática (ciclo de Cori) y fuente energética para el músculo cardíaco y fibras oxidativas del músculo estriado esquelético durante la contracción (Boffi, 2006); sin embargo, los hidrogeniones retenidos por el lactato son quienes producen el descenso del $\mathrm{pH}$ intracelular llevando a la acidosis láctica que genera finalmente la fatiga muscular (Boffi, 2006).

La concentración de LS por debajo del umbral anaeróbico varía mínimamente de la observada en reposo. A niveles submáximos de ejercicio, si no se sobrepasa el umbral, se apreciará un equilibrio entre la producción y eliminación existiendo un estado estable metabólico (García del Moral et al., 1998; Lindner, 2010). A medida que aumenta la intensidad del ejercicio la demanda de energía por unidad de tiempo supera la velocidad con la que puede ser producida en forma aeróbica, y los músculos son inducidos a producir energía en forma anaeróbica (Boffi, 2006), aumentando la cantidad de lactato, sobrepasando el punto de máximo estado estable del lactato en sangre (Lindner, 2010), comenzado a producirse un incremento no lineal (Ghosh, 2004). Este punto se conoce en humanos como umbral de lactato, y equivale a una concentración en sangre de $4 \mathrm{mmol} / \mathrm{l}$ de lactato (García del Moral et al., 1998; Ghosh, 2004; Lindner, 2010). La detección de dicha concentración es utilizada como punto final para pruebas de ejercicio (Kittleson et al., 1996), y predice de forma eficaz el rendimiento aeróbico (Ghosh, 2004; McArdle et al., 2004; Lindner, 2010). Se dispone de varios trabajos en equinos y humanos sobre el tema; sin embargo, son escasos en caninos (Yoshida et al., 1987; Gondim et al., 2007; Lindner, 2010).

En un programa de entrenamiento de resistencia se presentan adaptaciones celulares en el transcurso de las semanas que aumentarán la velocidad de eliminación del lactato, de forma que solo se acumulará a intensidades muy altas de ejercicio (McArdle et al., 2004). Es por ello que deportistas entrenados acumulan menores cantidades de lactato a cargas submáximas de trabajo (Ghosh, 2004). Por lo tanto, para mantener la eficacia del entrenamiento será necesario aumentar la carga impuesta a medida que mejora el rendimiento; lo cual se verá reflejado en la FC, que ante un esfuerzo regular a una potencia determinada sufrirá una disminución progresiva (Stepien et al., 1998; Grandjean et al., 2000).

\section{Cinta Trotadora Motorizada: Su Aplica- ción en el Perro Deportista}

La cinta trotadora motorizada ha sido utilizada como una herramienta para el análi- 
sis de la marcha en humanos, equinos y caninos (Fanchon y Grandjean, 2009), así como también para evaluar el efecto del entrenamiento sobre el organismo del perro (Piccione et al., 2012) y durante pruebas de esfuerzo (Kittleson et al., 1996; Ferasin y Marcora, 2007, 2009). Su uso permite precisar una carga de trabajo de intensidad (velocidad), duración y grado de inclinación suficientes como para producir un efecto mensurable; facilitando así, la estandarización de pruebas de esfuerzo y de programas de entrenamiento (Ferasin y Marcora, 2009).

Por otra parte, se ha reportado que la adaptación previa de los animales al uso de la cinta permite obtener resultados más confiables (Fanchon y Grandjean, 2009). Si bien faltan estudios sobre rutinas de entrenamiento específicas, su aplicación tiene un lugar prometedor para maximizar el rendimiento deportivo en el perro.

\section{Adaptaciones del Organismo Canino al Entrenamiento Deportivo}

El entrenamiento genera en el organismo diversas modificaciones metabólicas del sistema cardiovascular y respiratorio, y demás efectos orgánicos y funcionales que incluyen el aparato musculoesquelético y equilibrio hormonal, entre otros (Grandjean et al., 2000). Se ha demostrado en el perro que los cambios fisiológicos y sanguíneos inducidos por el esfuerzo, dependen de la duración e intensidad del ejercicio así como del nivel de entrenamiento previo del animal (Piccione $e t$ $a l ., 2012)$. Al iniciar una actividad, la función cardiovascular aumenta para satisfacer las necesidades musculares de sustratos energéticos y favorecer la eliminación de deshechos.

Tanto en los seres humanos como en los perros, el entrenamiento deportivo ha sido asociado con aumentos del volumen plasmático (Stepien et al., 1998). Así, los animales entrenados desarrollan adaptaciones que maximizan el trabajo cardíaco. La dismi- nución de la FC en reposo junto con la hipertrofia ventricular izquierda es el resultado del entrenamiento físico (Stepien et al., 1998). De hecho, la presencia de bradicardia en reposo es frecuentemente utilizada para confirmar que el entrenamiento ha sido eficaz (Billman y Kukielka, 2007).

Los trabajos de resistencia aumentan significativamente la densidad capilar y el tamaño y número de mitocondrias (McArdle et al., 2004). También provocan un aumento en la actividad de las enzimas mitocondriales, lo que mejora el rendimiento por mayor oxidación de las grasas y disminución de la producción de lactato (Ghosh, 2004). Todo esto potencia la capacidad celular para generar energía de forma aeróbica (McArdle et al., 2004). Por otra parte, la ventilación pulmonar prácticamente no se modifica, pero se obtiene una disminución de la frecuencia respiratoria debida a una mejor eficacia ventilatoria global (Grandjean et al., 2000).

\section{Nutrición Orientada al Deporte}

La nutrición en caninos de deporte tiene como objetivo minimizar las posibles lesiones debidas al ejercicio (deshidratación, hipertermia, mioglobinuria, lesiones músculo esqueléticas), así como maximizar el rendimiento (velocidad, fuerza, resistencia) (Hill, 1998). Afortunadamente se cuenta con diversos estudios sobre las necesidades nutricionales del perro de deporte (Kronfeld et al., 1994; Hill, 1998; Grandjean et al., 2000), que resultan de gran ayuda para la formulación de la dieta.

Como norma general, una dieta en perros deportistas debe ser hiperdigestible, garantizar el mejor aporte energético y contribuir a optimizar los procesos de detoxificación (Grandjean et al., 2000). Los requerimientos energéticos deben ajustarse en cantidad y calidad en relación al trabajo muscular y adaptarlos a la evolución del entrenamiento 
(Grandjean et al., 2000). En ejercicios de resistencia, se produce un aumento significativo de los ácidos grasos libres plasmáticos a partir de la degradación de triglicéridos almacenados en el tejido celular subcutáneo (Boffi, 2006). Esto mejora los procesos de oxidación de las grasas, favoreciendo la utilización de los lípidos presentes en su alimentación (Grandjean et al., 2000).

Se ha demostrado en caninos que la cantidad de energía obtenida por la oxidación de las grasas, tanto en reposo como durante el esfuerzo, es el doble que en especies menos aeróbicas como seres humanos y cabras (Hill, 1998). Existe mayor afinidad de la albúmina plasmática por los ácidos grasos libres en sangre, lo que mejora su llegada a los tejidos (Hill, 1998). De esta manera, en deportes de resistencia, al aumentar la oxidación de los ácidos grasos se evita la utilización de glucógeno muscular y glucosa sanguínea (Kronfeld et al., 1994), menos eficientes desde el punto de vista energético (McArdle et al., 2004).

Según la finalidad deportiva, se tienen necesidades nutricionales bien diferenciadas. Así, los perros de trineo requieren altas cantidades de grasa (más del 50\% de la energía) para aumentar la resistencia y maximizar la producción energética. Los valores proteicos también deben ser altos (más del 30\%) para evitar la anemia inducida por el ejercicio (Hill, 1998). Contrariamente, los galgos de carrera tienen mejor rendimiento cuando son alimentados con un moderado aumento de grasa en la ración $(31 \%)$ y valores más bajos de proteína (24\%) (Hill, 1998).

\section{Conclusiones}

- El perro de deporte necesita de una óptima preparación para poder lograr objetivos concretos. Para ello, es imprescindible reunir dos pilares fundamentales, el entrenamiento y la nutrición. El éxito deportivo no será posible si alguno de estos factores falla. Ambos deben ser adecuados y específicos a la finalidad deportiva que practica el animal.

- Se deben instaurar programas de entrenamiento y dietas que permitan maximizar el rendimiento en una determinada actividad, así como también evitar las patologías relacionadas al ejercicio. La medición de LS se muestra como un método factible para valorar la respuesta al esfuerzo y planificar las rutinas convenientes al propósito del perro. El uso de la cinta trotadora puede facilitar la realización de pruebas de ejercicio así como el posterior entrenamiento del animal.

\section{Literatura Cittada}

1. Billman GE, Kukielka M. 2007. Effect of endurance exercise training on heart rate onset and heart rate recovery responses to submaximal exercise in animals susceptible to ventricular ûbrillation. J Appl Physiol 102: 231-240. doi: 10.1152/japplphysiol.00793.2006

2. Boffi FM. 2006. Metabolismos energéticos y ejercicio. En: Fisiología del ejercicio en equinos. Buenos Aires: InterMédica. p 3-13.

3. Date A, Simonson S, Ransdell L, Gao Y. 2013. Lactate response to different volume patterns of power clean. J Strength Cond Res 27: 604-610. doi: 10.1519/JSC.0b013e31825d9bd9

4. Fanchon L, Grandjean D. 2009. Habituation of healthy dogs to treadmill trotting: Repeatability assessment of vertical ground reaction force. Res Vet Sci 87:135-139.doi: 10.1016/j.rvsc.2008.11.001

5. Ferasin L, Dodkin SJ, Amodio A, Murray JK, Papasouliotis K. 2007. Evaluation of a portable lactate analyzer (Lactate Scout) in dogs. Vet Clin Pathol 36: 36-39.

6. Ferasin L, Marcora S. 2007. A pilot study to assess the feasibility of a submaximal exercise test to measure 
individual response to cardiac medication in dogs with acquired heart failure. Vet Res Commun 31: 725-737. doi: 10.1007/ s11259-007-3566-7

7. Ferasin L, Marcora S. 2009. Reliability of an incremental exercise test to evaluate acute blood lactate, heart rate and body temperature responses in Labrador retrievers. J Comp Physiol B 179: 839-845. doi: 10.1007/s00360-009-0367-z

8. García del Moral L, García del Moral J, Rodríguez Jerez A. 1988. Factores que afectan a la lactatemia durante un test de esfuerzo. Arch Med Deporte 5: 375-386.

9. Ghosh AK. 2004. Anaerobic threshold: its concept and role in endurance sport. Malays J Med Sci 11: 24-36.

10. Gondim FJ, Zoppi CC, Pereira Da Silva L, De Macedo DV. 2007. Determination of the anaerobic threshold and maximal lactate steady state speed in equines using the lactate minimum speed protocol. Comp Biochem Physiol A Mol Integr Physiol 146: 375-380. doi: 10.1016/j.cbpa.2006.11.002

11. Grandjean D, Moquet N, Pawlowiez $S$, Tourtebatte AK, Jean B, Bacqué $H$. 2000. Guía práctica del perro de deporte y de utilidad. Francia: Royal Canin. $415 \mathrm{p}$.

12. Hill RC. 1998. The nutritional requirements of exercising dogs. J Nutr 128: 2686S-2690S.

13. Kittleson MD, Johnson LE, Pion PD. 1996. Submaximal exercise testing using lactate threshold and venous oxygen tension as endpoints in normal dogs and in dogs with heart failure. J Vet Intern Med 10: 21-27.

14. Kronfeld DS, Ferrante PL, Grandjean D. 1994. Optimal nutrition for athletic performance, with emphasis on fat adaptation in dogs and horses. J Nutr 124: 2745S-2753S.

15. Lindner AE. 2010. Maximal lactate steady state during exercise in blood of horses. J Anim Sci 88: 2038-2044. doi: 10.2527/jas.2009-2693

16. McArdle WD, Katch FI, Katch V.L. 2004. Transferencia energética durante el ejercicio en el ser humano. En: Fundamentos de fisiología del ejercicio. $2^{\mathrm{a}}$ ed. Madrid: McGraw-Hill. p 127-146.

17. Palmer J. 1994. Breed groups. En: The illustrated encyclopedia of dog breeds. New Jersey: Wellfleet Press. p 16-19.

18. Piccione G, Casella S, Panzera M, Giannetto C, Fazio F. 2012. Effect of moderate treadmill exercise on some physiological parameters in untrained beagle dogs. Exp Anim 61: 511-515.

19. Stepien RL, Hinchcliff KW, Constable $P D$, Olson J. 1998. Effect of endurance training on cardiac morphology in Alaskan sled dogs. J Appl Physiol 85: 1368-1375.

20. Yoshida T, Chida M, Ichioka M, Suda Y. 1987. Blood lactate parameters related to aerobic capacity and endurance performance. Eur J Appl Physiol Occup Physiol 56: 7-11. 